\title{
Aspectos da Funcionalidade Psicológica em Crianças e Adolescentes com Sobrepeso e Obesidade
}

\author{
Fernando Oliveira Pereira ${ }^{1}$ \\ Universidade Lusófona de Lisboa - Lisboa, Portugal
}

\begin{abstract}
Resumo: Sobrepeso e obesidade provêm do comportamento alimentar, determinado por fatores inerentes à funcionalidade do sistema bio-psico-social integrado. O objectivo consistiu no estudo de aspectos da funcionalidade psicológica em crianças e adolescentes com sobrepeso e obesidade. Investigação quantitativa de tipo quasi-experimental e abordagem comparativa entre dois grupos: experimental e controle. Amostra de 50 crianças e adolescentes dos 7 aos 18 anos com sobrepeso/obesidade, e outros 50 , com características sociodemográficas equivalentes, constituintesdo grupo de controle. Instrumentos metodológicos: Entrevista; questionário sociodemográfico; inventário de transtornos alimentares. Os resultados revelam que o grupo com sobrepeso/obesidade, comparativamente aos do grupo de controle, apresenta valores mais expressivos, com diferenças estatisticamente significativas, de IMC e da tríade de transtornos alimentares: obsessão pelo emagrecimento, bulimia, insatisfação corporal; das características psicológicas "sentimento de ineficácia", e menos na "consciência interoceptiva" e "medo da maturidade". Conclui-se, em situação de avaliação, que os participantes com sobrepeso e obesidade evidenciam, simultâneamente, impulsos para o emagrecimento e ingestão alimentar excessiva, confirmando a perturbação do comportamento alimentar, onde impera a conflitualidade interior entre a consciência da necessidade de evitar a ingestão alimentar excessiva e a dificuldade em controlar o impulso de atração sensorial para a ingestão alimentar; quando excessiva pode desencadear atitudes de insatisfação corporal e esta vir a ser uma componente negativa da imagem corporal. A funcionalidade psíquica é dominada por sentimentos de ineficácia, consciência interoceptiva insuficiente e menor receio da maturidade, propiciando sentimentos de inadequação e insegurança face ao controlo do comportamento alimentar.
\end{abstract}

Palavras-chave: aspectos psicológicos, sobrepeso, obesidade infantil, atitudes alimentares

\section{Aspects of Psychological Functionality in Children and Adolescents with Overweight and Obesity}

\begin{abstract}
Overweight and obesity arise from eating behavior, determined by factors inherent to the functionality of the integrated bio-psycho-social system. The objective consisted of the study of aspects of psychological functionality in overweight and obese children and adolescents. Quantitative quasi-experimental investigation and comparative approach between two groups: experimental and control. A sample of 50 overweight/ obese children and adolescents from 7 to 18 years old, and another 50, with equivalent sociodemographic characteristics, constituted the control group. Methodological instruments: Interview; sociodemographic questionnaire; inventory of eating disorders. The results reveal that the overweight/obese group, compared to the control group, presents more expressive values, with statistically significant differences, of BMI and the triad of eating disorders: obsession with weight loss, bulimia, body dissatisfaction; of the psychological characteristics "feeling of ineffectiveness", and less in "interoceptive awareness" and "fear of maturity". It is concluded, in an evaluation situation, that overweight and obese participants, simultaneously, show impulses for to lose weight and impulse excessive food intake, confirming the disturbance in eating behavior, where internal conflicts prevail of between the awareness of the need to avoid excessive food intake and the difficulty in controlling the sensory attraction impulse for food intake; when excessive, it can trigger attitudes of body dissatisfaction and this becomes a negative component of body image. The psychic functionality is dominated by feelings of inefficience, insufficient interoceptive awareness, and less fear of maturity, leading to feelings of inadequacy and insecurity regarding the control of eating behavior.
\end{abstract}

Keywords:psychological aspects, overweight, childhood obesity, eating attitudes

1 Doutor em Psicologia; Professor Coordenador; Escola Superior de Educação Almeida Garrett/ Universidade Lusófona de Lisboa - Portugal.E-mail: fopereira@sapo.pt

Submetido em: 16/03/2021. Primeira decisão editorial: 22/04/2021. Aceito em: 13/05/2021. 


\section{Introdução}

O sujeito com sobrepeso ou obesidade é um sistema bio-psico-social, integrado de múltiplos fatores de naturezas diversas, cuja complexidade estrutural e funcional opera a vários níveis e dimensões, constituindo-se ainda de aspectos e componentes (Ganzen, 1984; Lomov, 1984; Morin, 1994; Pereira, 2018).

As causas do sobrepeso e da obesidade provêm de uma variedade de fatores: genéticos, ambientais, condições sociais, pressões económicas, estilos de vida familiares ou de outros problemas de saúde. Existem outras causas modificáveis, que potenciam o excesso de peso; uma das mais relevantes é os comportamentos alimentares pouco saudáveis, sendo aqueles em que há exageros ou exclusão de alimentos. Nos saudáveis passa-se o inverso, sem exageros e sem exclusão de alimentos. Os hábitos alimentares e o sedentarismo são os maiores responsáveis pela progressão da prevalência da obesidade infantil; por isso, na prevenção deverse-á incidir na promoção da alimentação saudável e na actividade física (Associação Brasileira para o Estudo da Obesidade [ABESO], 2020; Ahrens \& Pigeot, 2015; Ministério da Saúde, 2019; World Health Organization [WHO], 2020).

\section{Prevalência}

A obesidade infantil e adulta tem aumentado tanto que é considerada uma epidemia e um problema de saúde pública (Freitas, 2014; Frontzek, 2015; Melo et al., 2003, Nunes et al., 2006; Reis et al., 2011).

A obesidade é um dos mais graves problemas de saúde, estimando-se que em 2025 haja 2,3 biliões de adultos no mundo com excesso de peso e 700 milhões de indivíduos com obesidade (WHO, 2020). Em 2017, a Organização Mundial de Saúde realizou um estudo que estimava existirem 124 milhões de crianças e adolescentes obesos em todo o mundo; em quatro décadas, o número de crianças e adolescentes com obesidade subiu exponencialmente de 11 milhões para 124 milhões. Além disso, há mais 123 milhões de crianças, adolescentes e jovens com idades compreendidas entre 5 e 19 anos com excesso de peso (Ministério da Educação, 2021; WHO, 2020). Em 2018, o Ministério da Saúde e a Organização Panamericana da Saúde registaram que $12,9 \%$ das crianças brasileiras entre os 5 e os 9 anos de idade e $7 \%$ dos adolescentes entre os 12 e os 17 anos têm obesidade (Ministério da Saúde, 2019).

A obesidade infantil é um dos principais desafios de saúde pública do séc. XXI no mundo; em particular porque nas crianças com excesso de peso ou obesidade existe maior probabilidade de permanecerem obesas na idade adulta e de desenvolver diabetes ou doenças cardiovasculares, numa idade ainda jovem (WHO, 2020).

No entanto, nos últimos tempos, com base em intervenções orientadas para a mudança de condições nutricionais, hábitos e estilos alimentares mais adequados tem vindo a registar-se diminuições, ainda que relativas, na prevalência da obesidade infantil (Alvarenga et al., 2015; Alvarenga et al., 2021). Tendência que também é verificada em Portugal, tal como na Europa, no decurso apenas de uma década, segundo os dados da 5 a fase do COSI Portugal (Sistema de Vigilância Nutricional Infantil do Ministério da Saúde) assistiu-se à diminuição do excesso de peso nas crianças, sendo que em 2008 eram 37,9\% e em 2019 eram 29,6\%. Embora, - COSI (Childhood Obesity Surveillance Initiative), confirme a diminuição do excesso de peso e da obesidade infantil em Portugal, não pode descurar a necessidade de manter a preocupação de vigilância dos hábitos alimentares no país; há registros que $40 \%$ dos adolescentes bebe refrigerantes diariamente, metade consome produtos hortofrutícolas abaixo do recomendável e mais de $20 \%$ consome açúcar acima dos níveis recomendados. Os dados da mesma organização revelam que a prevalência da obesidade infantil aumentou com a idade: $15,3 \%$ das crianças de 8 anos são obesas, incluindo $5,4 \%$ com obesidade severa; valor que é de $10,8 \%$ em crianças de 6 anos ( $2,7 \%$ obesidade severa) (SNS, 2019).

\section{Etiologia}

Atualmente, existe unanimidade que a obesidade é uma doença crônica multifatorial, 
envolvendo aspectos genéticos, psicológicos, sociais, culturais e ambientais, cujas comorbilidades afectam a qualidade de vida e implicam riscos para a saúde dos pacientes; por isso, a abordagem terapêutica também deve assentar numa perspetiva multidisciplinar. $\mathrm{Na}$ obesidade a relação entre os vários elementos que a configuram é dinâmica e simultânea; daí que abordagens terapêuticas fragmentadas correm o risco de serem pouco eficazes (ABESO, 2015, 2020; Moraes et al., 2014). A complexidade das patologias alimentares requer uma intervenção que envolve a articulação de várias áreas do conhecimento, nomeadamente a medicina, a nutrição, a psicologia, a psicoterapia. A interdisciplinaridade constitui a articulação de várias disciplinas focadas no objeto da intervenção. Portanto, a abordagem interdisciplinar reporta-se à convocação de diferentes disciplinas ou especialidades de intervenção em conformidade com a singularidade do objeto e de cada caso (Minayo, 2010, 2011; Farias et al., 2017).

O comportamento alimentar que conduz ao excesso de peso e à obesidade frequentemente provém de influências mediáticas, as quais promovem o consumo de alimentos inadequados, do marketing e da publicidade que estimulam o consumismo, dos interesses da indústria de alimentos processados; isto é válido para crianças, adolescentes e adultos. Contudo, os padrões ideais de magreza, a complexidade das relações familiares envolvidas, a discriminação social, as dificuldades subjetivas de cada um, entre outros, reportam-se às faixas etárias da adolescência, juventude e idade adulta (Frontzek, 2015; Frontzek et al., 2017; Nunes et al., 2006).

A obesidade além de problema de saúde pública, também é uma questão social, devido ao estigma atribuído às pessoas com excesso de peso, podendo afetar negativamente a autoestima e incrementar a tendência para o isolamento social (Poulain, 2013). Em 5\% dos casos a obesidade infantojuvenil é endógena, decorrendo de fatores genéticos e neuroendócrinos, mas em $95 \%$ dos casos a origem é exógena, determinada por fatores externos de ordem variada: desmame afetivo precoce, rotinas sedentárias e atividade física escassa, alimentação nutricional hipercalórica e dinâmicas familiares disfuncionais (Carvalho et al., 2013). Estima-se que $25 \%$ das crianças e $80 \%$ dos adolescentes com excesso de peso ou obesidade mantêm-se obesas na idade adulta (Marchi-Alves et al., 2011).

Consequências psicossociais da obesidade na infância e adolescência. A obesidade tem consequências negativas de natureza psicossocial na vida dos indivíduos, podendo afetar a autoestima, em função da estigmatização, da dificuldade de aceitação da autoimagem corporal, do sentimento de fracasso, de inferioridade e da vivência de situações de bullying (Melo et al., 2011). O impacto da obesidade na saúde mental está relacionado com alterações emocionais de ansiedade, depressão, irritabilidade, medo, raiva, tristeza, autoestima, imagem corporal negativa e sofrimento psíquico associado ao bullying, níveis significativos de stresse, timidez, medos excessivos e rejeição social, provocando baixa autoestima (Andrade et al., 2014; Turco et al., 2013), autoconceito negativo decorrente da perceção negativa de si e da estigmatização física advindas de brincadeiras depreciativas (Assunção et al. 2013).

A sociedade ao instituir padrões de imagem corporal ideal faz com que surjam estigmas de se ser "gordo", sentindo-se as crianças inferiorizadas face a outras; daí a obesidade poder ser vista como condição para a estigmatização, exclusão e discriminação social (Simões \& Menezes, 2007; Ricciardelli \& MacCabe, 2001; Wardl \& Cooke, 2005; Latner et al., 2005).

A insatisfação com a imagem corporal e o bullying na adolescência estão frequentemente associados à obesidade (Crivelaro \& Tuma, 2015; Scutti et al., 2014). A perceção e atitude das crianças face a terem excesso de peso, em particular devido a serem alvo de bullying na escola e em casa, interferem na sua vida quotidiana, no desenvolvimento de atividades físicas, nas relações sociais e na saúde mental (Borges, Barreto et al., 2018).

Os problemas de saúde mental nas pessoas com obesidade são predominantemente o stresse, a ansiedade e a depressão, os quais resultam da insatisfação corporal, perceção da autoimagem negativa, baixa autoestima e associação pejorativa face aos padrões de estética corporal (Melca \& 
Fortes, 2014). Embora os sintomas depressivos e de ansiedade sejam os prevalecentes, em crianças e adolescentes com excesso de peso, outros aspectos de natureza psicológica e psicossocial, tais como o temperamento, o stresse, coping mal adaptativo e contextos de risco psicossocial são igualmente fatores de influência negativa (Bellodi, 2018).

O sedentarismo é um comportamento recorrente nos adolescentes, sendo um fator que contribui para a reduzida queima de calorias e acumulação de gordura corporal devido às rotinas diárias que passam por défice de atividades físicas e domínio de atividades como ver televisão e videojogos. Também a falta de apoio familiar e o baixo suporte afetivo e emocional podem interferir negativamente com o processo terapêutico e de afrontamento psicossocial da obesidade, devido à dificuldade dos pais exercerem controle efetivo sobre o comportamento alimentar dos filhos (Nogueira \& Zambon, 2013; Rocha et al., 2017).

A literatura reporta a dificuldade dos familiares compreenderem a obesidade nos filhos adolescentes, por eles mesmos, pais e avós, padecerem de quadro semelhante, tornando-se prejudicial na adoção de estratégias para enfrentar o problema; daí ser recomendável implementar medidas de educação para saúde com intuito de alcançar mudanças dos padrões familiares (Andrade et al., 2014; Rocha et al., 2017; Turco et al., 2013).

No âmbito da intervenção psicológica, em vez de estratégias de controle e prevenção, através de programas prescritivos, verticais e culpabilizantes, propõe-se ações de prevenção baseadas na lógica de responsabilidade partilhada (Poulain, 2013).

Um aspecto possível de influenciar negativamente os tratamentos na obesidade infantil é a perceção distorcida dos pais sobre o excesso de peso dos seus filhos (Frontzek, 2016). As intervenções que não envolvem os pais são menos eficazes (Camargo et al., 2013; Melo et al., 2003). É importante envolver o conjunto familiar, escutar os filhos e os pais e compreender as relações estabelecidas no sistema familiar, porque o comportamento alimentar é aprendido. As narrativas das crianças não são muito diferentes das dos pais. Há tendência para a repetição da obesidade entre gerações, sendo fatores determinantes neste processo o ambiente familiar e os hábitos alimentares da família (Coradini et al., 2017).

A análise de conteúdo é uma técnica instrumental que visa o agrupamento de conteúdos provenientes de materiais de diversa ordem, como por exemplo discursos, comunicações, textos, com base em critérios previamente delineados, permitindo construir classificações, nas quais são formadas categorias que estruturam e enquadram possíveis interpretações dos fatos. A análise de conteúdo das narrativas das crianças com excesso de peso e dos seus pais permitiu construir cinco categorias temáticas: significado da comida, perceção social das pessoas com excesso de peso, influência do excesso de peso infantil no quotidiano, a relação com profissionais da saúde e os motivos para a não adesão ao tratamento (Frontzek et al., 2017).

\section{Tratamento}

O tratamento com base em abordagens terapêuticas diferenciadas permite implementar formas de intervenção mais consentâneas às especificidades do objeto ao qual são dirigidas. Por isso, para compreender a obesidade infantil e pensar intervenções mais eficazes deve-se considerar o sentido atribuído à obesidade, as relações familiares, as condições socioeconómicas e todos os elementos que circundam o fenómeno; deve-se focar na pessoa e não na obesidade (Frontzek et al., 2017).

A obesidade infantil poderá vir a assumir contornos de cronicidade, devido ao acumular excessivo de massa adiposa, mas também devido à acção de outros fatores, desde a idade de bebé até aos 12 anos, acarretando prejuízos para a saúde; visto que o excesso de peso provoca transtornos que, independentemente de serem, ou não, fatais, comprometem a qualidade de vida futura. $\mathrm{O}$ excesso de peso na idade escolar tende a estender-se ao longo do processo de desenvolvimento, tendo consequências nefastas para a saúde na idade adulta (Souza, 2019).

A obesidade infantil está ligada a uma multiplicidade de aspectos e fatores; em alguns deles as crianças passam a maior parte do seu dia a dia a ver televisão ou outros ecrãs eletrónicos, o sedentarismo e a consumir alimentos ultraprocessados, muitas 
das vezes devido a influência dos pais ou a fatores socioeconómicos (Souza, 2019).

Existem vários métodos ou instrumentos para medir a massa corporal, mas a antropometria é um dos métodos mais aplicados na prática clínica, porque não é dispendioso, nem invasivo, disponível para ter acesso ao peso e à composição do corpo humano, refletindo fatores tão importantes como a saúde, a nutrição, o risco e a sobrevivência (Rolland-Cachera, 2002; Weiss et al., 2016). Um dos indicadores mais razoáveis é o IMC (Índice de Massa Corporal), o qual tem sido associado ao aumento de pressão arterial, ao perfil de suscetibilidade à lipoproteína, diabetes não dependentes de insulina e lesões de arterosclerose (Dietz \& Robinson, 1998). Estudos sobre o crescimento mostraram que rapazes e raparigas com sobrepeso registaram aumento de risco de morbilidade associada à obesidade comparativamente a adolescentes magros (Must et al., 1992). Outro estudo que acompanhou crianças do nascimento aos 21 anos de idade, demonstrou que o RA (Recuperação da Adiposidade) precoce se encontra associado a maior IMC e à dobra cutânea subescapular na idade de 21 anos (Araujo et al., 2018; Dumith \& Junior, 2010; Rolland-Cachera et al., 1987; Viveiro et al., 2016).

O sucesso na abordagem do excesso de peso/obesidade, aquando da implementação de novas estratégias de intervenção, depende dos conhecimentos detidos acerca dos determinantes comportamentais e psicossociais dos sujeitos a quem se dirige a ação (Vaz et al., 2010).

As atitudes e comportamentos alimentares das pessoas resultam do processo de socialização e desenvolvimento. Tradicionalmente são aprendidas no seio da família, sofrem influência dos pares, das experiências de cidadania, dos conhecimentos obtidos na escola e informação veiculada pelos meios de comunicação social (Viana et al., 2003). O ato de comer é um processo biológico, mas é a cultura que determina a forma como os seres humanos se comportam e fazem as suas escolhas alimentares (Kiess, Marcus \& Wabitsch, 2004; Viana et al., 2009). O que se come e a quantidade de alimentos ingeridos é influenciado pelo património cultural, atitudes e práticas familiares (Savage et al., 2007).
Contudo, existem alterações do comportamento alimentar na infância que decorrem de dificuldades em se integrarem no meio social, apresentando manifestações do foro psicológico como ansiedade, depressão, baixa autoestima, distorção da perceção da imagem corporal e outros (Alvarenga et al., 2021; Dixon et al., 2003; Moschonis et al., 2008; Luiz et al., 2005).

As crianças e adolescentes com ou em risco de terem transtornos alimentares revelam atitudes inadequadas à ingestão e restrição alimentar; por exemplo, episódios de ingestão compulsiva e comportamentos compensatórios devido à incapacidade para controlar e gerir adequadamente o processo de ingestão alimentar (Ricciardelli \& McCabe, 2001; Galindo \& Carvalho, 2007; Decaluwé \& Braet, 2005; Ximenes, 2019).

Os determinantes do comportamento alimentar não assentam exclusivamente em mecanismos endógenos de natureza biológica, sendo importante a compreensão da experiência subjetiva do apetite e as preferências por alimentos específicos. A regulação do apetite está associada ao estado de saciedade do sujeito, havendo participação tanto de mecanismos endógenos de natureza biológica como de natureza psicológica e também de mecanismos exógenos de natureza ambiental (Sinopoulou et al., 2015). Existe estreita relação entre o apetite e a saciedade, visível na análise da selecção de alimentos e bebidas, motivação para ingerir, preferências e desejos individuais, consumo calórico, envolvendo as sensações de fome e saciedade, assim como diversos fatores de ordem cognitiva e emocional no processo de regulação do apetite; assim o evidencia o esquema e a descrição da cascata da saciedade, resultando em parte significativa do padrão recorrente do comportamento alimentar (Blundell et al., 1993; Sinopoulou et al., 2015).

As estratégias de intervenção no âmbito da obesidade infantil não se devem limitar à mudança de hábitos alimentares, devendo ser um processo dinâmico de construção de um novo estilo de vida, levando sempre em linha de conta a saúde, o bemestar físico e psicológico e que a compreensão do desenvolvimento do apetite na infância esclarece quais são os caminhos que conduzem ao ganho de 
peso ao longo da vida (Santos et al., 2016; Sinopoulou et al., 2015; Vaz et al., 2010). Por conseguinte, o objetivo da investigação prende-se com o estudo de aspectos da funcionalidade psicológica em crianças e adolescentes portadores de sobrepeso e obesidade. Os aspectos ou especificidades psicológicas são elementos estruturantes que orientam e condicionam a funcionalidade psíquica do ser humano. Nesta investigação os aspectos psicológicos visados pelo estudo são os definidos pelo instrumento metodológico utilizado, o qual incorpora características relativas ao transtorno do comportamento alimentar e características que enformam a funcionalidade psíquica em termos de sentimentos de ineficácia, orientação para o perfecionismo, desconfiança interpessoal, consciência interoceptiva, medo de enfrentar as demandas da vida adulta, ascetismo, impulsividade e insegurança social.

\section{Método}

\section{Amostra e Tipo de Estudo}

Amostra constituída por crianças e adolescentes, com idades compreendidas entre os 7 e os 18 anos, referenciados por médicos de medicina geral e familiar ou por pediatras à consulta de pediatria do âmbito da obesidade por terem excesso de peso, o qual em parte dos participantes se enquadra na obesidade infantil; por isso, passaram a ter acompanhamento médico especializado em clínicas situadas na Região de Saúde de Lisboa e Vale do Tejo, com a finalidade de controlar os fatores que eventualmente pudessem estar na origem do problema. A faixa etária da população é aquela que se enquadra na idade pediátrica, que recorria à consulta especializada estritamente por questões de sobrepeso ou obesidade, e por se tratar de crianças e adolescentes que já tinham competências de leitura e escrita suficientes para poderem responder aos questionários. Contudo, principalmente aos mais novos, foi dispensado apoio para assegurar que compreendiam as perguntas e superar dificuldades que surgissem a cada momento.

Os participantes do grupo de controle foram selecionados aleatoriamente desde que cumprissem os seguintes critérios: Não ter excesso de peso; ter idade, escolaridade e outras caraterísticas sociodemográficas idênticas às do grupo experimental.

As questões éticas foram salvaguardadas recolhendo consentimento informado e esclarecido referente a cada participante individualmente junto do próprio, dos seus progenitores e do médico que o seguia na consulta; no caso do grupo de controle o consentimento envolveu o participante e os seus progenitores.

\section{Participantes}

Tabela 1

Caraterização sociodemográfica e condição clínica dos participantes

\begin{tabular}{|c|c|c|c|c|}
\hline \multicolumn{2}{|c|}{ Caraterísticas } & 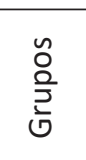 & \multirow{2}{*}{$\begin{array}{r}\begin{array}{c}\text { Diagnóstico } \\
\text { de sobrepeso } \\
\text { / obesidade }\end{array} \\
50 \\
\end{array}$} & \multirow{2}{*}{$\begin{array}{r}\begin{array}{l}\text { Grupo de } \\
\text { controle }\end{array} \\
50\end{array}$} \\
\hline \multirow{3}{*}{ 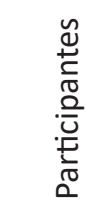 } & \multicolumn{2}{|c|}{ Total } & & \\
\hline & \multicolumn{2}{|c|}{$\begin{array}{l}\text { Sexo } \\
\text { masculino }\end{array}$} & $19(38 \%)$ & $18(36 \%)$ \\
\hline & \multicolumn{2}{|c|}{ Sexo feminino } & $31(62 \%)$ & 32 (64\%) \\
\hline \multicolumn{3}{|c|}{ Intervalo etário } & $7-18$ anos & $8-18$ anos \\
\hline \multirow{3}{*}{. } & \multicolumn{2}{|c|}{$7-10$ anos } & $25(50 \%)$ & $12(24 \%)$ \\
\hline & \multicolumn{2}{|c|}{$10-14$ anos } & $20(40 \%)$ & $20(40 \%)$ \\
\hline & \multicolumn{2}{|c|}{$14-18$ anos } & $5(10 \%)$ & $18(36 \%)$ \\
\hline \multicolumn{3}{|c|}{ Estado Civil: solteiro } & $50(100 \%)$ & $50(100 \%)$ \\
\hline \multirow{4}{*}{$\begin{array}{l}\frac{0}{0} \\
\frac{\pi}{0} \\
\frac{0}{L} \\
\frac{\pi}{0} \\
\\
\tilde{U} \\
.\end{array}$} & \multicolumn{2}{|c|}{$\begin{array}{l}\text { 10 ciclo: } 2^{\circ}, 3^{\circ} \\
\text { e 40 ano }\end{array}$} & $21(42 \%)$ & $12(24 \%)$ \\
\hline & \multicolumn{2}{|c|}{$\begin{array}{l}20 \text { ciclo: } 5 \% \text { e } \\
60 \text { ano }\end{array}$} & $10(20 \%)$ & $14(28 \%)$ \\
\hline & \multicolumn{2}{|c|}{$\begin{array}{l}\text { 3ㅇ ciclo: } 70,80 \\
\text { e 9o ano }\end{array}$} & $18(36 \%)$ & $16(32 \%)$ \\
\hline & \multicolumn{2}{|c|}{$\begin{array}{l}\text { Secundário: } \\
10^{\circ}, 11^{\circ} \text { e } 12 \text { } \\
\text { ano }\end{array}$} & $1(2 \%)$ & $8(16 \%)$ \\
\hline \multirow{6}{*}{ 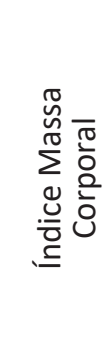 } & \multicolumn{2}{|c|}{ Intervalo IMC } & $\begin{array}{r}22,60- \\
40,34 \\
\end{array}$ & $18,5-26,30$ \\
\hline & \multicolumn{2}{|c|}{ IMC $18-20$} & $0(0 \%)$ & $34(68 \%)$ \\
\hline & \multicolumn{2}{|c|}{ IMC 20-25 } & $2(4 \%)$ & $12(24 \%)$ \\
\hline & \multicolumn{2}{|c|}{ IMC 25-30 } & $32(64 \%)$ & $4(8 \%)$ \\
\hline & \multicolumn{2}{|c|}{ IMC $30-35$} & $12(24 \%)$ & $0(0 \%)$ \\
\hline & \multicolumn{2}{|c|}{ IMC $35-40$} & $4(8 \%)$ & $0(0 \%)$ \\
\hline
\end{tabular}




\section{Caraterização e Tipo de Estudo}

A investigação consistiu num estudo quantitativo de tipologia quasi-experimental com a implementação de abordagem comparativa entre dois grupos: o experimental e o de controle.

O objetivo da investigação prende-se com o estudo de aspectos da funcionalidade psicológica em crianças e adolescentes com sobrepeso e obesidade, tendo motivado a colocação da seguinte hipótese de trabalho - existem aspectos ou especificidades da funcionalidade psíquica que diferenciam crianças e adolescentes com diagnóstico de sobrepeso ou obesidade daqueles que não comportam esse mesmo diagnóstico.

\section{Delineamento da Pesquisa}

No período de tempo entre março e junho de 2020 foram identificados e referenciados os participantes da pesquisa. Depois disto, no mesmo período, procedeu-se ao contato com os participantes e respetivos progenitores para obtenção do consentimento informado e esclarecido. Os dados foram coletados no período que medeia entre junho e novembro de 2020 em horários variados de acordo com a disponibilidade das clínicas, dos participantes e dos seus progenitores. Os instrumentos metodológicos foram aplicados individualmente e na presença do investigador, o qual se manteve em permanente interação comunicativa com o participante desde o inicio até ao final da sessão, esclarecendo as instruções, questões em que o participante evidenciava dúvidas de compreensão e nas escalas de resposta em termos de objetivação de valores.

Embora a pesquisa tivesse sido desenvolvida em tempo de pandemia do coronavírus, foi assegurado que os efeitos da pandemia e as alterações das rotinas de vida dos participantes não tiveram implicações significativas nos procedimentos e nos resultados da pesquisa. Os próprios relataram na entrevista que não houve mudanças significativas no seu estilo de vida e não se sentiram afetados, tendo mantido atividade física, apesar de esta ter sido realizada noutras condições de acordo com as recomendações de proteção e defesa face à situação pandémica.

\section{Critérios de Inclusão e Exclusão}

Os critérios de inclusão que orientam a seleção dos participantes na pesquisa consistem em ter diagnóstico de sobrepeso ou obesidade, IMC correspondente ao diagnóstico, idades compreendidas entre 7 e 18 anos, não padecer de outras patologias, em particular do foro psiquiátrico, no grupo experimental, e ausência de sobrepeso ou obesidade e de outras patologias, IMC dentro dos parâmetros da norma, no grupo de controle.

\section{Procedimentos}

Depois de terem sido explicadas, ao participante, as etapas do processo de avaliação dá-se inicio à sessão. Em primeiro lugar foi aplicado o questionário sociodemográfico, procedendo-se à recolha dos dados sociodemográficos. A seguir foi aplicado o inventário de transtornos alimentares (EDI - Eating Disorder Inventory), instruindo o participante a dar uma resposta de acordo com a escala de frequência com que aquele fenómeno se apresenta em si. Por fim, é encetada uma entrevista semi-estruturada com o participante orientada para a obtenção de informações de natureza clínica, psicológica e psicossocial acerca de si com a finalidade de ajudar a compreender melhor algo que não tivesse quedado claro nas respostas aos questionários.

\section{Instrumentos Metodológicos}

\section{Cálculo do Índice de Massa Corporal (IMC)}

Com base nas medidas de peso em quilogramas e estatura em metros, efetuadas em todos os participantes, foi calculado o Índice de Massa Corporal (IMC) através da aplicação da fórmula de Quetelet: IMC = Kg/m² (Garrow \& Webster, 1985). No entanto, por se tratar de crianças e adolescentes foram tidos em consideração os valores determinados 
por três critérios de referência baseados no índice de massa corporal (IMC): Center Disease Control and Prevention (CDC), Organização Mundial de Saúde (OMS), International Obesity Task Force (IOTF) (Cole et al., 2000; Cole et al., 2007; Onis et al., 2007; Weiss et al., 2016), sendo que tratando-se de população portuguesa foram seguidas as recomendações da Direção Geral de Saúde (DGS), utilizando as curvas de percentis de IMC do CDC, incluídos no Boletim de Saúde Infantil e Juvenil (BSIJ) português (Ministério da Saúde, 2013; Viveiro et al., 2016).

Tabela 2

Pontos de corte de percentis $(P)$ de índice de massa corporal (IMC) para classificação de crianças e adolescentes em conformidade com as curvas de referência do $C D C$, OMS e IOTF

\begin{tabular}{l|r|r|r}
\hline \multirow{2}{*}{$\begin{array}{l}\text { Valores } \\
\text { Classificação }\end{array}$} & \multicolumn{3}{c}{ Percentis de IMC } \\
\hline Baixo peso & $\mathrm{IMC}<\mathrm{P5}$ & $\mathrm{IMC}<\mathrm{P3}$ & $\begin{array}{r}\mathrm{IMC}< \\
17 \mathrm{Kg} / \mathrm{m}^{2}\end{array}$ \\
\hline Peso normal & $\mathrm{P} 5<\mathrm{IMC}<$ & $\mathrm{P} 3<\mathrm{IMC}<$ & $\begin{array}{r}17 \mathrm{Kg} / \mathrm{m}^{2} \\
<\mathrm{IMC}< \\
25 \mathrm{Kg} / \mathrm{m}^{2}\end{array}$ \\
\hline Sobrepeso & $\mathrm{P} 85<\mathrm{IMC}<$ & $\mathrm{P} 85<\mathrm{IMC}<$ & $\begin{array}{r}25 \mathrm{Kg} / \mathrm{m}^{2} \\
<\mathrm{IMC}< \\
30 \mathrm{Kg} / \mathrm{m}^{2}\end{array}$ \\
\hline Obesidade & $\mathrm{IMC}>\mathrm{P} 95$ & $\mathrm{IM}>\mathrm{P} 97$ & $\begin{array}{r}\mathrm{IMC}> \\
30 \mathrm{Kg} / \mathrm{m}^{2}\end{array}$ \\
\hline
\end{tabular}

CDC - National Center for Chronic Disease Prevention and Health Promotion; OMS - Organização Mundial de Saúde; IOTF - International Obesity Task Force; P Percentil; IMC - Índice de Massa Corporal

\section{Entrevista Clínico-Psicológica}

Entrevista de tipologia clínica no formato semiestruturado orientada aos aspectos psicológicos relacionados com a funcionalidade do sujeito nas diversas dimensões da sua vida. A utilização da entrevista neste formato tem a finalidade de obter informação relevante para a investigação e de aprofundar especificidades da funcionalidade psíquica, familiar, social e comunitária do participante face ao comportamento alimentar e também de colmatar algumas das insuficiências, caraterísticas de instrumentos estruturados como o são os questionários fechados.

\section{Questionário Sócio-demográfico}

Questões orientadas para recolha de informação sobre categorias como idade, escolaridade, condição familiar, peso e estatura.

\section{Inventário de Transtornos Alimentares (EDI - Ea- ting Disorder Inventory)}

O Inventário de Transtornos Alimentares consiste num questionário baseado no autorelato, sendo frequentemente usado para avaliar a presença de transtornos alimentares relacionados com as anorexia e bulimia nervosas, mas também do transtorno alimentar não especificado de outras formas, incluindo o transtorno de compulsão alimentar periódica.

O questionário original, criado em 1984, por David M. Garner e M. P. Olmsted, era constituído por 64 questões, distribuídas por oito subescalas. Cada questão é submetida a avaliação pelo respondente numa escala de 6 pontos, variando de "Nunca" a "Sempre".

As 8 subescalas do EDI são:

1. Impulso para emagrecer ou obsessão pelo emagrecimento - revela preocupação em fazer dieta, preocupação com o peso e medo de ganhar peso;

2. Bulimia - tendência a ter pensamentos e atrações pela comida; episódios de compulsão alimentar;

3. Insatisfação corporal - revela insatisfação com o seu corpo e com a aparência física, sendo uma componente relacionada com a perturbação da imagem corporal;

4. Ineficácia-avalia sentimentos de inadequação, insegurança, inutilidade, incapacidade de controle sobre as suas vidas;

5. Perfecionismo - não ficar satisfeito com nada que não seja perfeito, orientação para alcançar níveis de rendimento perfecionistas;

6. Desconfiança interpessoal - relutância em formar relacionamentos íntimos, dificuldade 
em expressar os seus sentimentos e pensamentos, necessidade de manter distância dos outros;

7. Consciência Interoceptiva - mede a habilidade do indivíduo para discriminar entre sensações e sentimentos e entre as sensações de fome e saciedade, revela a dificuldade em responder adequadamente aos estados emocionais;

8. Medo da Maturidade - medo de enfrentar as demandas da vida adulta, revela desejo de se sentir seguro como na infância.

Mais tarde, o próprio autor procedeu a duas revisões, das quais resultaram o Eating Disorder Inventory - 2 (EDI - 2) (Garner et al., 1991; Garner, 1998) e o Eating Disorder Inventory - 3 (EDI - 3) (Garner, 2010).

A versão da revisão em 1991 consistiu no Eating Disorder Inventory - 2 (EDI - 2), usado em homens e mulheres acima dos 12 anos, mantendo o que era do inventário original e incluindo mais 27 questões novas, as quais passaram a formar mais três escalas:

a) Ascetismo - tendência para procurar a virtude por meio de certos ideais espirituais, tais como a autodisciplina, o sacrifício, autosuperação e controle das necessidades corporais;

b) Impulsividade - avalia a dificuldade para regular os impulsos e as tendências, mostra a capacidade de regular o comportamento impulsivo, especialmente o comportamento compulsivo;

c) Insegurança Social - revela medos sociais e a insegurança, mede as crenças sobre as relações sociais inseguras, incómodas e insatisfatórias.

A última revisão, datada de 2004; deu origem ao Eating Disorder Inventory - 3 (EDI - 3), o qual é constituído pelas questões das versões 1 e 2, tendo sido aperfeiçoado para responder às teorias mais modernas relacionadas com 0 diagnóstico de transtornos alimentares e é composto por 91 questões, distribuídas por 12 subescalas, usando o sistema de pontuação de 0 a 4 pontos para cada questão. Três subescalas reportam-se especificamente aos transtornos alimentares e nove a aspectos psicológicos relevantes para os transtornos alimentares.

Contudo, apesar do EDI - 2 estar orientado para faixas etárias acima dos 12 anos e este estudo incluir crianças em idade escolar a partir dos 7 anos, optou-se pelo uso do EDI - 2, tendo-se procedido a certas adaptações semânticas e de interpretação das questões de modo a assegurar que as crianças a partir dos 7 anos compreendiam e interpretavam adequadamente o que liam. Entretanto, antes de iniciar a investigação, mas já sabendo quem eram os participantes, foi confirmado que eles se encontravam num nível de domínio da leitura e consequente compreensão dos conteúdos, assegurando que estavam em condições de responderem ao respetivo questionário. Além disso, o investigador esteve presente, todo o tempo em que o participante respondia ao questionário, individualmente, disponibilizandose para esclarecer, orientar e apoiar sempre que se verificasse necessário e oportuno. Não obstante a utilização de um instrumento que não estava propriamente aferido para uma parte das idades dos participantes, foram criadas as condições suficientes para eliminar possíveis obstáculos à compreensão das questões do questionário.

\section{Resultados}

$\mathrm{Na}$ tabela 3 que se segue são inscritos os valores obtidos pelo grupo de sobrepeso/ obesidade infantil e pelo grupo de controle. Os valores inscritos reportam-se ao índice de massa corporal (IMC) e às caraterísticas da funcionalidade psíquica resultantes da aplicação do inventário de transtornos alimentares (EDI - 2, Eating Disorder Inventory), providenciando adaptações semânticas e de interpretação e assegurando as condições necessárias para que os participantes, especificamente desta investigação, em idade escolar acima dos 7 anos, pudessem responder adequadamente ao questionário. 
Tabela 3

Diferenças nas especificidades de funcionalidade psíquica em crianças e adolescentes com sobrepeso/obesidade e do grupo de controle.

\begin{tabular}{|c|c|c|c|c|c|c|c|}
\hline \multirow{2}{*}{$\begin{array}{c}\text { Grupos } \\
\text { Categorias }\end{array}$} & \multicolumn{2}{|c|}{$\begin{array}{l}\text { Sobrepeso/ } \\
\text { Obesidade }\end{array}$} & \multicolumn{2}{|c|}{ Grupo de Controle } & \multirow{2}{*}{ 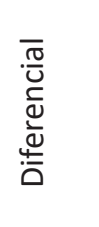 } & \multirow{2}{*}{ 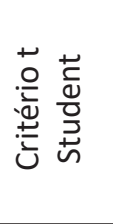 } & \multirow{2}{*}{ 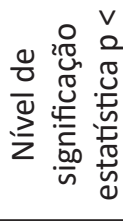 } \\
\hline & M & DP & $\mathrm{M}$ & $\mathrm{DP}$ & & & \\
\hline Índice de Massa Corporal & 29,21 & 3,87 & 19,60 & 2,93 & 9,60 & 14,95 & 0,000 \\
\hline Obsessão pelo emagrecimento & 16,50 & 7,22 & 11,96 & 8,93 & 4,54 & 2,92 & 0,005 \\
\hline Bulimia & 10,08 & 5,21 & 7,82 & 5,87 & 2,26 & 1,93 & 0,059 \\
\hline Insatisfação corporal & 24,04 & 8,35 & 15,94 & 12,20 & 8,10 & 3,71 & 0,001 \\
\hline Sentimento de ineficácia ineficiência & 17,58 & 8,09 & 12,56 & 8,43 & 5,02 & 2,88 & 0,006 \\
\hline Perfecionismo & 14,52 & 4,83 & 13,44 & 6,21 & 1,08 & 1,01 & 0,316 \\
\hline Desconfiança interpessoal & 15,32 & 6,94 & 14,74 & 6,48 & 0,58 & 0,42 & 0,673 \\
\hline Consciência interoceptiva & 17,18 & 6,61 & 19,72 & 8,43 & $-2,54$ & $-1,72$ & 0,091 \\
\hline Medo da maturidade & 19,22 & 5,85 & 21,72 & 7,79 & $-2,50$ & $-1,88$ & 0,066 \\
\hline Ascetismo & 13,48 & 5,57 & 11,86 & 6,32 & 1,62 & 1,28 & 0,205 \\
\hline Impulsividade & 19,54 & 10,12 & 17,30 & 9,98 & 2,24 & 1,09 & 0,278 \\
\hline Insegurança social & 15,38 & 6,13 & 12,50 & 9,18 & 2,88 & 1,61 & 0,113 \\
\hline
\end{tabular}

Dos resultados inscritos na tabela 3 verifica-se que o índice de massa corporal (IMC) entre o grupo de participantes com diagnóstico de sobrepeso e obesidade e o grupo de controle, constituído por participantes, os quais são referência da norma, apresenta diferenças estatisticamente significativas $(p<0.000)$, sendo os valores bastante mais expressivos quando os participantes têm sobrepeso ou obesidade. Salienta-se que os resultados são relativos ao grupo de crianças e adolescentes juntos, não se tendo optado por formar dois grupos separados. É importante especificar que, de acordo com os referenciais do IMC, no grupo de sobrepeso/obesidade cerca de $50 \%$ dos participantes enquadram-se no diagnóstico de sobrepeso e outros $50 \%$ no diagnóstico de obesidade, se atendermos a que 18\% apresenta IMC entre 29 e 30; daí poder-se incorporar na obesidade, juntando aos $32 \%$ com valores claramente acima de 30.

Nas três primeiras escalas, a seguir à que representa o IMC, consideradas pelo autor do EDI (Garner, $1984,1998)$, relacionadas com os transtornos alimentares propriamente ditos e que são a obsessão pelo emagrecimento, a bulimia e a insatisfação corporal evidenciam-se diferenças estatisticamente significativas entre os dois grupos de participantes da investigação ( $p<0.005,0.05,0.001$, respetivamente); pertencendo os valores de maior expressividade ao grupo de sobrepeso/obesidade.

Nas outras oito escalas seguintes, que o autor considera estarem orientadas para revelar alterações em determinados aspectos ou caraterísticas psicológicas, diferenças estatisticamente significativas existem na escala "sentimento de ineficácia" ( $p<0.006$ ) com maior expressividade no grupo de sobrepeso/obesidade e nas escalas "consciência interoceptiva" e "medo da maturidade" ( $<<0.09$ e $p<0.06$, respetivamente) com maior expressividade no grupo de controle, ainda que as diferenças estatísticas se encontrem ao nível de tendência.

Nas escalas relacionadas com perfecionismo, desconfiança interpessoal, ascetismo, impulsividade e insegurança social não foram encontradas diferenças estatisticamente significativas, mas há maior expressividade de valores no grupo de sobrepeso e obesidade. No entanto, no que respeita às escalas de impulsividade e insegurança social, fica-se com a impressão que, apesar das diferenças de médias ser expressiva, as diferenças estatísticas não alcançaram o patamar de significância porque existe elevado grau de dispersão dos valores como mostra o desvio-padrão. 


\section{Discussão}

Nesta investigação o índice de massa corporal (IMC) apresenta-se claramente como um referencial de diagnóstico importante; primeiro na diferenciação de crianças e adolescentes com sobrepeso e obesidade e, em segundo lugar, como indicador da possível existência de inadequações do comportamento alimentar. No grupo experimental, visado pela investigação como podendo apresentar problemas do foro do comportamento alimentar, todos os participantes tinham $\mid \mathrm{MC}>25, \mathrm{P}>85$, excepto 2 casos que se encontravam no limiar já muito próximo do valor referido para a condição de sobrepeso. Por conseguinte, cerca de $32 \%$ das crianças e adolescentes apresentaram IMC> 30, $\mathrm{P}>95$, e se a estes acrescentar $18 \%$ dos participantes cujo IMC se insere no intervalo [29,5 - 29,9], então totalizará $50 \%$ de crianças e adolescentes em que o diagnóstico provável é de obesidade. Os restantes $50 \%$ de crianças e adolescentes do grupo experimental apresentavam IMC $>25$ e $<30$, P $>85$ e $\mathrm{P}<95$, dominando os valores do espectro [27 - 29]; sendo o diagnóstico provável de sobrepeso (Ahrens \& Pigeot, 2015; Cole et al., 2000; Cole et al., 2007, Viveiro et al., 2016).

0 recurso às informações recolhidas no âmbito da entrevista e observação clínicopsicológica, aos pais e aos filhos, inferem que todos os participantes do grupo com sobrepeso/obesidade tinham comportamento alimentar desadequado da sua condição etária, estilo e rotina alimentar diária, tendo em conta as suas necessidades nutricionais e a realidade da sua vida. Dados confirmados nas entrevistas realizadas aos pais e aos filhos e também pelos profissionais de saúde que acompanhavam os participantes nas consultas de controle do peso e orientação alimentar. São frequentes as queixas dos pais sobre comportamentos dos filhos como comer às escondidas, repetir a ração, comendo em demasia à refeição, ao longo do dia devoram pacotes de bolachas, preferências por alimentos ricos em gorduras e hidratos de carbono; chegando a amuar e até ofender quem se recusa a permitir que leve avante as suas intenções alimentares, dizendo, por vezes com raiva e ódio "queres é que eu passe fome, matar-me à fome", sendo que os filhos corroboraram estes comportamentos em entrevistas individuais e serem habituais na sua rotina diária (Frontzek, 2015; Frontzek et al., 2017; Nunes et al., 2006).

Muitas vezes a discórdia alimentar entre pais e filhos é motivo para gerar tensão emocional nas relações interpessoais e na funcionalidade familiar. No entanto, há casos em que o conflito por causa do comportamento alimentar dos filhos não existe, até porque os pais consideram que é normal, que eles também eram assim e estão bem; que o querer controlar mais os alimentos e a quantidade ingerida é excesso de zelo dos profissionais de saúde (Andrade et al., 2014; Nogueira \& Zambon, 2013; Rocha et al., 2017; Turco et al., 2013).

De acordo com as investigações de vários autores, tendo em conta as teorias do comportamento alimentar, o índice de massa corporal correlaciona positivamente com a dimensão "Restrição Alimentar" (Pirke \& Laessel, 1993). Significa quando há um IMC mais elevado a tendência é para existirem comportamentos orientados para a restrição alimentar; provavelmente por se tomar consciência em determinada fase da vida que tem cometido erros e exageros do foro alimentar e que deverá adotar medidas para corrigir os seus hábitos e estilo alimentar (Camargo et al., 2013; Coradini et al., 2017; Frontzek, 2016; Frontzek et al., 2017; Melo et al., 2003; Poulain, 2013).

Nas três primeiras escalas, que na tabela 3 são as que se seguem à inscrição do IMC, consideradas pelo autor do EDI (Garner \& Olmsted, 1984; Garner, 1998), relacionadas com os transtornos alimentares propriamente ditos, reportando-se às dimensões: obsessão pelo emagrecimento, bulimia e insatisfação corporal, evidenciam-se diferenças estatisticamente significativas entre os dois grupos de participantes da investigação ( $p<0.005,0.05$, 0.001 , respetivamente); pertencendo os valores de maior expressividade ao grupo de sobrepeso/ obesidade. Significa que as crianças e adolescentes portadoras de sobrepeso ou obesidade, no momento da avaliação, reconhecem ter atitudes que evidenciam posições e atitudes relacionadas com o emagrecimento, as quais certamente representam a preocupação com o excesso de peso atual; por 
isso, no plano psicológico o comportamento de resposta é gerado por ideias e pensamentos que denotam interesse orientado para perder peso, ou seja emagrecer (Alvarenga et al., 2021; Sinopoulou et al., 2015). Contudo, paralelamente e ao mesmo tempo, os valores mais altos obtidos na escala de bulimia indiciam a existência de tendências para a excessiva ingestão alimentar, sentindo atração, por vezes de difícil superação, pela comida (Decaluwé \& Braet, 2005; Galindo \& Carvalho, 2007; Ricciardelli \& McCabe, 2001; Ximenes, 2019). Além disso, estas crianças e adolescentes revelam atitudes de insatisfação corporal; portanto, com os contornos físicos do seu corpo; daí a revelação de ideias para perder peso (Alvarenga et al., 2021; Bellodi, 2018; Melca \& Fortes, 2014). A insatisfação corporal é apresentada como um dos aspectos que constituem a estrutura psicológica da perturbação da imagem corporal (Garner, 1998; Ximenes, 2019); entretanto, nas entrevistas clínico-psicológicas realizadas não se verifica que no dia a dia os participantes revelem preocupações significativas com o fato de terem excesso de peso; essa preocupação é mais dos médicos assistentes e dos pais. Então, a preocupação evidenciada pelos participantes no momento da avaliação parece ser mais o reflexo situacional das circunstâncias em que se vê envolvido; fato que é facilmente verificável ao longo das sessões de acompanhamento clínico e psicológico em que há dificuldade no cumprimento das regras e dos conselhos nutricionais e dietéticos sugeridos (Alvarenga et al., 2015; Alvarenga et al., 2021).

Nas outras oito escalas seguintes, que o autor considera estarem orientadas para revelar alterações em determinados aspectos ou caraterísticas psicológicas, diferenças estatisticamente significativas existem na escala relativa à dimensão "sentimento de ineficácia" ( $p<0.006)$, com maior expressividade no grupo de sobrepeso/obesidade e nas escalas relativas às dimensões "consciência interoceptiva" e "medo da maturidade" ( $p<0.09$ e $p<0.06$, respetivamente) com maior expressividade no grupo de controle, apesar do grau de significatividade das diferenças estatísticas se encontrar ao nível de tendência. Diferenças que demonstram a maior tendência das crianças e adolescentes portadores de sobrepeso e obesidade para experimentarem sentimentos de inadequação e insegurança, no que concerne a controlar os seus comportamentos, incapacidade para diferenciarem interiormente sensações de sentimentos e entre sensações de fome e saciedade e ainda para não se sentirem preocupados com a ideia de alcançarem a maturidade antes de tempo; visto não se sentirem inseguros socialmente na condição em que se encontram e, por isso, não necessitam procurar segurança nos períodos anteriores da sua vida (Dixon et al., 2003; Moschonis et al., 2008; Luiz et al., 2005; Ximenes, 2019).

Nas escalas relacionadas com as dimensões: perfecionismo, desconfiança interpessoal, ascetismo, impulsividade e insegurança social, não foram encontrados diferenças estatisticamente significativas, mas há maior expressividade de valores no grupo de sobrepeso e obesidade. No entanto, no que respeita às escalas de impulsividade e insegurança social, fica-se com a impressão que apesar das diferenças de médias ser expressiva as diferenças estatísticas não alcançaram o patamar de significância porque existe elevado grau de dispersão dos valores como mostra o desvio-padrão. Fato que evidenciaria a tendência das crianças e adolescentes com sobrepeso e obesidade para serem mais impulsivas, devido à menor capacidade de controlar e regular conscientemente o seu comportamento, sentindo-se também menos seguras no âmbito dos seus contatos e relações sociais. Por conseguinte, é provável que estes participantes não mostrem preocupações relativamente ao perfecionismo, visto que nos seus relatos não almejam alcançar maior rendimento pessoal do que aquele que os carateriza. No que respeita à impulsividade parece que de fato há dificuldade, em parte devido à falta de motivação e vontade, para se esforçarem no controle dos impulsos orientados para a ingestão alimentar, cedendo frequentemente às suas atrações sensoriais e pretensões baseadas na funcionalidade cognitivoemocional (Alvarenga et al., 2021; Blundell et al., 1993; Sinopoulou et al., 2015). A provável tendência relativa à insegurança social, talvez resulte na possível existência de relações sociais tensas, inseguras e incómodas, devido à pressão que se exerce sobre ele para mudar o estilo de vida alimentar no seio 
do círculo familiar, sob orientação clínica, mas que o próprio provavelmente não está ainda disposto a fazê-lo, sentindo-se desagradado com as frequentes chamadas de atenção para os cuidados a ter em termos de comportamento alimentar. É importante salientar que a pressão exercida sobre as crianças e adolescentes, participantes da pesquisa, para modificarem a relação com a comida não advém das suas próprias motivações, mas sim da parte dos seus pais e profissionais de saúde, os quais consideram que o excesso de peso resultante de atitudes e comportamento alimentar dos portadores pode ser nefasto para a sua condição de saúde.

A interpretação dos resultados obtidos infere que na abordagem dos problemas relacionados com sobrepeso e obesidade infantil assumem importância incalculável o papel desempenhado pela psicoeducação, aconselhamento psicológico e psicoterapia, nomeadamente a cognitivocomportamental, visando o comportamento alimentar através da mudança de representações e atitudes (Andrade et al., 2014; Frontzek et al., 2017; Garner \& Garfinkel, 1997; OPP, 2014; Rocha et al., 2017; Turco et al., 2013). Saliente-se que as intervenções de âmbito terapêutico devem ser sustentadas em abordagens teóricas de cariz sistémico-integrativo em que o ser humano seja sempre mais importante que o corpo e que a comida seja sempre valorizada antes do nutriente (Alvarenga et al., 2015; Pereira, 2018); porquanto deva envolver toda a família e os que comportam o ambiente nuclear circundante a mudar o seu estilo de vida e relação com a comida.

\section{Conclusão}

Todos os participantes com diagnóstico de sobrepeso/obesidade apresentam índice de massa corporal em conformidade e a maior parte deles não evidencia preocupação com esse indicador.

Os participantes com sobrepeso e obesidade apresentam valores significativamente expressivos na tríade de escalas relacionadas com o índice de perturbações alimentares: impulso para o emagrecimento, bulimia e insatisfação corporal. A maior expressividade em simultâneo nas escalas de impulso para o emagrecimento e de impulso para a ingestão alimentar indicia a existência de conflitualidade interior, pelo menos no âmbito da avaliação situacional, em que os participantes tomam consciência da necessidade de evitar a ingestão alimentar excessiva para perder peso e, ao mesmo tempo, reconhecem, por um lado, a existência, em si, de impulsos para a ingestão alimentar excessiva e, por outro lado, a dificuldade em controlar o impulso de atração sensorial que motiva a excessiva ingestão alimentar. No plano da representação psicológica a condição de ingestão alimentar excessiva conduz frequentemente a situações que desencadeiam atitudes de insatisfação corporal, as quais habitualmente e de acordo com os padrões culturais vigentes na pessoa em questão contribuem em maior ou menor grau para a instalação de imagem corporal negativa. Explicação válida para as faixas etárias da adolescência e juventude em que os padrões socioculturais relacionados com a estética corporal são uma componente importante na construção da sua imagem corporal. Na infância o que leva a insatisfação corporal a contribuir para a imagem corporal negativa não são os padrões da estética cultural, mas sim as avaliações decorrentes das apreciações depreciativas feitas pelos pais e pares a quem as crianças querem agradar.

A funcionalidade psíquica no plano cognitivoemocional dos participantes com sobrepeso e obesidade é dominada por sentimentos de ineficácia mais expressivos e consciência interoceptiva insuficiente e menor receio da maturidade, demonstrando tendência para a revelação de sentimentos de inadequação e insegurança, no que concerne a controlar os seus comportamentos, nomeadamente o alimentar, evidenciando incapacidade ou, porventura, dificuldade em discriminar interiormente, com a clareza necessária, sensações de sentimentos; assim como também em estabelecer diferenciações precisas entre sensações de fome e de saciedade.

Os resultados obtidos pelos participantescom sobrepeso e obesidade nas escalas: perfecionismo, desconfiança interpessoal, ascetismo, impulsividade e insegurança social, indiciam que nalguns deles a funcionalidade psíquica é comprometida pela 
presença de caraterísticas de impulsividade e insegurança social, evidenciando no controle e regulação consciente do seu comportamento, sentindo-se, então, menos seguras no âmbito dos seus contatos e relações sociais.

Nos participantes com sobrepeso e obesidade a impulsividade, no que concerne ao comportamento alimentar, em parte é devido à falta de motivação e vontade para se esforçarem no controle dos impulsos orientados para a ingestão alimentar, cedendo frequentemente às suas atrações sensoriais e pretensões baseadas na funcionalidade cognitivo-emocional. A insegurança social provavelmente decorre de relações sociais tensas, inseguras e incómodas, devido à pressão que se exerce sobre ele para mudar o estilo de vida alimentar no seio do círculo familiar, sob orientação clínica, mas o próprio não estar ainda disposto ou preparado psicologicamente para a mudança.

$\mathrm{Na}$ abordagem multidisciplinar de crianças e adolescentes com sobrepeso e obesidade assume importância estratégica medidas de âmbito psicoeducacional, aconselhamento psicológico e da terapia cognitivo-comportamental orientada à mudança de representações e atitudes face ao comportamento alimentar. Preconizam-se intervenções de âmbito sistémico-integrativo, adotando estratégias dirigidas ao ser humano como um todo integrado em vez de se restringir apenas a questões de índole corporal, envolvendo no processo terapêutico a família e todos os sistemas relacionais do ambiente nuclear circundante significativo, visando a mudança de atitudes e relações para alcançar a correção de aspectos da funcionalidade psicológica de modo a proporcionar condições que assegurem modificações efetivas do estilo de vida e comportamento alimentar.

\section{Contribuição}

O pesquisador declara não haver conflitos de interesse.

\section{Referências}

Associação Brasileira para o Estudo da Obesidade e da Síndrome Metabólica. (2015). Mapa da obesidade. Abeso. http://www.abeso.org.br/ atitude-saudavel/mapa-da-obesidade

Associação Brasileira para o Estudo da Obesidade e da Síndrome Metabólica. (2020). Mapa da obesidade. https://www.abeso.org.br/obesidadee-sindrome-metabolica/mapa-da-obesidade

Associação Brasileira para o Estudo da Obesidade e da Síndrome Metabólica. (2020). Os últimos números da obesidade no Brasil. https:// www.abeso.org.br/os-ultimos-numeros-daobesidade-no-brasil

Ahrens, W. \& Pigeot, I. (2015). Fatores de risco da obesidade infantil: Lições do Estudo Europeu IDEFICS. In M. L. Frelut (Ed.), The ECOG's eBook on Child and Adolescent Obesity. ebook.ecogobesity.eu

Alvarenga, M., Figueiredo, M., Timerman, F. \& Antonaccio, C. (2015). Nutrição comportamental. Manole.

Alvarenga, M. S., Dunker, K. L. L. \& Philippi, S. T. (Orgs.). (2021). Transtornos alimentares e nutrição: da prevenção ao tratamento. Manole.

Andrade, T. D. M., Moraes, D. E. B. D., \& AnconaLopez, F. (2014). Problemas Psicológicos e Psicodinâmicos de crianças e adolescentes obesos: relato de pesquisa. Psicologia: Ciência e Profissão, 34, 126-141. doi: 10.1590/S141498932014000100010.

Araujo, M. L. D., Andrade, M. L. S. S., Prado, L. V. S., Cabral, S. P., Cabral, P. C. \& Grande de Arruda, I. K. (2018). Precisão do IMC em diagnosticar o excesso de gordura corporal avaliada pela bioimpedância elétrica em universitários. Nutrición Clínica Dietética Hospitalaria, 38(3), 154-160. DOI: 10.12873/383diniz.

Assunção, M. C. F., Muniz, L. C., Schäfer, A. A., Meller, F. D. O., Carús, J. P., Quadros, L. D. C. M. D., \& Menezes, A. M. B. (2013). Tornar-se obeso na adolescência pode trazer consequências à saúde mental? Caderno de Saúde Pública, 29, 1859-1866. Doi: 10.1590/0102-311X00135812 
Bellodi, A. C. (2018). Obesidade em crianças e adolescentes: temperamento, estresse, coping e risco psicossocial familiar [Tese de Doutorato, Pontifícia Universidade Católica de Campinas].

Blundell, J. E., Burley, V. J., Cotton, J. R., \& Lawton, C. L. (1993). Dietary-Fat and the Control of Energy-Intake: Evaluating the Effects of Fat on Meal Size and Postmeal Satiety. American Journal of Clinical Nutrition, 57(5), S772-S8.

Borges, F., Barreto, M.S., Reis, P., Viera, C. S. \& Marcon, S. S. (2018). Percepções e atitudes de crianças que vivenciam a obesidade. Rene, 19. DOI: 10.15253/2175-6783.2018193261 www. revistarene.ufc.br

Ministério da Educação. (n.d.). Obesidade infantil é tema do programa Salto para o Futuro. Recuperado em 27 abril, de http://portal.mec. gov.br/component/tags/tag/obesidade-infantil

Ministério da Saúde. (2019). Vigitel Brasil 2018: vigilância de fatores de risco e proteção para doenças crónicas por inquérito telefónico. Ministèrio da Saúde.

Camargo, A. P. P. M., Azevedo, B. A. \& Antonio, M. A. R. M. (2013). A não percepção da obesidade pode ser um obstáculo no papel das mães de cuidar de seus filhos. Ciência e Saúde Coletiva, 18(2), 323-333.

Carvalho, E. A. D. A., Simão, M. T. J., Fonseca, M. C., Andrade, R. G. D., Ferreira, M. S. G., Silva, A. F. \& Fernandes, B. S. (2013). Obesidade: aspectos epidemiológicos e prevenção. Revista Médica de Minas Gerais, 23, 74-82. DOI: 10.5935/22383182.20130012.

Cole, T. J., Bellizzi, M. C., Flegal, K. M. \& Dietz, W. H. (2000). Establishing a standard defintion for child overweight and obesity worldwide: International survey. BMJ, 320(7244), 1240-3.

Cole, T. J., Flegal, K. M., Nicholls, D. \& Jackson, A. A. (2007). Body mass índex cut offs to define thinness in children and adolescents: International survey. BMJ, 335(7612), 166-167.

Coradini, A. O., Moré, C. L. O. O. \& Scherer, A. A. (2017). Obesidade, família e transgeracionalidade: uma revisão integrativa da literatura. Nova Perspectiva Sistêmica, 58, 17-37.
Crivelaro, S. H. R., \& Tuma, M. Â. F. (2015). Nível de atividade física, obesidade e bullying em escolares. Corpo e Movimento Educação Física, 6, 29-35.

Decaluwé, V. \& Braet, C. (2005). The cognitive behavioural model for eating disorders: A direct evaluation in children and adolescents with obesity. Eating Behaviors, 6(3), 211-20.

Dietz, W. H. \& Robinson, T. N. (1998). Use of the body mass index (BMI) as a measure of overweight in children and adolescents. $J$. Pediatr, 132(2), 191-3.

Dixon, J. B., Dixon, M. E., \& O’Brien, P. E. (2003). Depression in Association with Severe Obesity. American Medical Association, 163(17), 205865.

Dumith, S. C. \& Junior, J. C. (2010). Sobrepeso e obesidade em crianças e adolescentes: comparação de três critérios de classificação baseados no índice de massa corporal. Revista Panaericana de Salud Publica, 28, 30-35.

Farias, C. P., Quintana, A. M. \& Olesiak, L. R. (2017). Patologias alimentares: um desafio interdisciplinar. Contextos Clínicos, 10(2), 197-208. https://dx.doi.org/10.4013/ ctc. 2017.102 .05

Freitas, F. C. (2014). A janela da escuta. Relato de experiência clinica. Scriptum.

Frontzek, L. G. M. (Org) (2015). Obesidades e psicologia. Juruá.

Frontzek, L. G. M. (2016). Obesidade infantil: Compreensão das relações familiares e intervenção psicossocial [Tese de Doutorado, Fundação Oswaldo Cruz/Centro de Pesquisa René Rachou]. www.arca.fiocruz. $\mathrm{br} /$ bitstream/icict/18027/2/Tese_CHSS_ LucianaGaudioMartinsFrontzek.pdf

Frontzek, L. G. M., Bernardes, L. R. \& Modena, C. M. (2017). Obesidade infantil: Compreender para melhor intervir. Abordagem Gestaltica, 23(2), 167-174.

Galindo, E. M., \& Carvalho, A. M. (2007). Translation, adaptation and internal consistency evaluation of the Eating Behaviours and Body Image Test for female children. Revista de Nutrição, 20(1), 47-54. 
Ganzen, V. A. (1984). Descrições sistémicas em psicologia. Universidade Estatal de Leninegrado.

Garner, D. M. (1998). Inventário de trastornos de la conducta alimentar (EDI-2). TEA Ediciones.

Garner, D. M. (2010). Inventário de trastornos de la conducta alimentar: Manual EDI-3. TEA Ediciones.

Garner, D. M. \& Garfinkel, P. E. (1982). Body image in anorexia nervosa: Measurement, theory and clinical implications. International Journal of Psychiatry in Medicine, 11(3), 263-284.

Garner, D. M. \& Garfinkel, P. E. (1997). Handbook of treatment for eating disorders. Guilford Press.

Garner, D. M. \& Olmsted, M. P. (1984). The Eating Disorder Inventory Manual. Psychological Assessment Resources.

Garner, D. M., Shafer, C. L. \& Rosen, L. W. (1991). Critical appraisal of the DSM-III-R diagnostic criteria for eating disorders. In S. R. Hooper, G. W. Hynd and R. E. Mattison (Eds.), Child psychopathology: Diagnostic criteria and clinical assessment: Current issues and procedures (vol. 1: Psychiatric disorders). Lawrence Erlbaum Associates.

Garrow, J., \& Webster, J. (1985). Quetelet index $(\mathrm{W} / \mathrm{H}$ ?) as a measure of fatness. International Journal of Obesity \& Related Metabolic Disorders, 9, 147-153.

Kiess, W., Marcus, C., \& Wabitsch, M. (2004). Obesity in Childhood and Adolescence. Pediatr Adolesc Medicine, 9, 124-36.

Latner, J. D., Stunkard, A., \& Wilson, G. T. (2005). Stigmatized Students: Age, Sex, and Etnicity Effects in the Stigmatization of Obesity. Obes Res, 13(17), 1226-31.

Lomov, B. F. (1984). Problemas teóricos e metodológicos da psicologia. Ciência.

Luiz, A. M., Gorayeb, R., Liberatore Júnior, R. R., \& Domingos, M. (2005). Depressão, ansiedade e competência social em crianças obesas. Estudos de Psicologia, vol. 10(3), 35-39.

Marchi-Alves, L. M., Yagui, C. M., Rodrigues, C. S., Mazzo, A., Rangel, E. M. L., \& Girão, F. B. (2011). Obesidade infantil ontem e hoje: importância da avaliação antropométrica pelo enfermeiro. Escola Anna Nery, 15(2), 238-244. doi: 10.1590/S1414-81452011000200004.
Melca, I. A., \& Fortes, S. (2014). Obesidade e transtornos mentais: construindo um cuidado efetivo. Revista Hospital Universitário Pedro Ernesto, 13(1), 18-25. DOI: 10.12957/ rhupe.2014.9794

Melo, E. D., Luft, V. C. \& Meyer, F. (2003). Obesidade Infantil: como podemos ser eficazes. Jornal de Pediatria, 80(3), 1-16.

Melo, T. R., Jansen, A. K., Pinto, R. D. M. C., Morales, R. R. D., Morales, N. M., Prado, M. M., \& Silva, C. H. M. D. (2011). Qualidade de vida de cuidadores de crianças e adolescentes com sobrepeso ou obesidade. Revista da Escola de Enfermagem USP, 45(2), 319-26. DOI: 10.1590/ S0080-62342011000200003

Minayo, M. C. 2010. Disciplinaridade, interdisciplinaridade complexidade. Emancipação, 10(1), 435-442. https://doi. org/10.5212/Emancipacao.v.10i2.435-442

Minayo, M. C. 2011. Da inteligência parcial ao pensamento complexo: desafios da ciência e da sociedade contemporânea. Política \& Sociedade, 10(19), 41-56.

Moraes, J. M., Caregnato, R. C. A. \& Schneider, D. S. (2014). Qualidade de vida antes e após a cirurgia bariátrica. Acta Paulista de Enfermagem, 27(2), 157-164. DOI: 10.1590/1982-0194201400028

Morin, E. (1994). La complexité humaine. Flammarion.

Moschonis, G., Grammatikaki, E., \& Manios, Y. (2008). Perinatal predictors of overweight at infancy and preschool childhood: the Genesis study. International Journal of Obesity, 32(1), 39-47.

Must, A., Jacques, P. F., Dallal, G. E., Bajema, C. J., \& Dietz, W. H. (1992). Long term morbidity and mortality of overweight adolescents - a follow-up of the Harvard group study of 1922 to 1935. New England Journal of Medicine, 327(19),1350-5.

Nogueira, T. F. D., \& Zambon, M. P. (2013). Reasons for non-adherence to obesity treatment in children and adolescents. Revista Paulista de Pediatria, 31(3), 338-343. DOI: 10.1590/S010305822013000300010. 
Nunes, M. A., Appolinario, J. C., Abuchaim, A. L. G. \& Coutinho W. (Eds). (2006). Transtornos alimentares e obesidade. Artmed.

Onis, M. D., Onyango, A. W., Borghi, E., Siyam, A., Nishida, C. \& Siekmann, J. (2007). Development of a WHO growth reference for school-aged children and adolescents. Bull World Health Organ, 85(9), 660-7.

Ordem dos Psicólogos Portugueses (OPP) (2014). Contributos da psicologia no excesso de peso, obesidade e perturbações alimentares. Ordem dos Psicólogos Portugueses.

Pereira, F. O. (2018). Teoria Sistémico-Integrativa do Psiquismo Humano. Teoría y Crítica de la Psicología, 10, 1-23. http://www.teocripsi.com/ ojs/(ISSN:2116-3480)

Pirke, K., \& Laessle, R. (1993). Restrained eating. In A. Stunkard, \& T. Wadden (Eds.), Obesity: Theory and therapy (2nd ed., pp. 151-162). Raven Press.

Ministério da Saúde (2013). Saúde infantile e juvenile: Programa-tipo de atuação. Direção Geral de Saúde. http://www.dgs.pt/?cr=24430

Poulain, J. P. (2013). Sociologia da obesidade. Senac.

Reis, C. E. G., Vasconcelos, I. A. L., \& Barros, J. D. N. (2011). Políticas públicas de nutrição para o controle da obesidade infantil. Revista Paulista de Pediatria, 29, 625-33. DOI: 10.1590/S010305822011000400024

Ricciardelli, L.A., \& MacCabe, M. P. (2001). Children's Body Image Concerns and Eating Disturbance: A Review of the Literature. Clinical Psychology Review, 21(3), 325-44.

Rocha, M., Pereira, H., Maia, R., Silva, E., Morais, N. \& Maia, E. (2017). Aspectos psicossociais da obesidade na infância e adolescência. Psicologia, Saúde \& Doenças, 18(3). DOI: http://dx.doi.org/10.15309/17psd1807

Rolland-Cachera, M. F., \& Cole, T. (2002). Measurements and definition. In W. Burniat, I. Lissau, \& T. Cole (Eds). The obese and overweight child (pp. 3-27). University Press.

Rolland-Cachera, M. F., Deheeger, M., Avons, P, Guilloud-Bataille, M., Patois, E., \& Sempé, M. (1987). Tracking adiposity patterns from 1 month to adulthood. Ann Hum Biol, 14, 219-22.
Santos, R. A., Maranhão, Th. L. G. \& Batista, H. M. T. (2016). Obesidade infantil e abordagens em psicologia: uma revisão da literatura. Revista Multidisciplinar e de Psicologia, 10(30), Supl. 1. http://idonline.emnuvens.com.br/id

Savage, J. S, Fisher, J. O., \& Birch, L. L. (2007). Parental Influence on Eating Behavior: Conception to Adolescence. Journal of Law Med Ethics, 35(1), 22-34.

Scutti, C. S., Seo, G. Y., Amadeu, R. S., \& Sampaio, R. F. (2014). O enfrentamento do adolescente obeso: a insatisfação com a imagem corporal e o bullying. Revista da Faculdade de Ciências Médicas de Sorocaba, 16, 130-133.

Simões, D. \& Meneses, R. F. (2007). Autoconceito em crianças com e sem obesidade. Psicologia: Reflexão e Crítica, 20(2), 246-251. http://www. scielo.br/pdf/prc/v20n2/a10v20n2.pdf

Sinopoulou, V., Harrold, J. \& Halford, J. (2015). Significado e análise da saciedade na infância. In M. L. Frelut (Ed.), The ECOG 's eBook on Child and Adolescent Obesity. ebook.ecog-obesity.eu

Serviço Nacional de Saúde (2019, 7 julho).

Portugal: obesidade infantil. sns.gov.pt/ noticias/2019/07/10/Portugal-obesidade-infantil-2

Souza, J. C. (2019). Fatores que contribuem para o desenvolvimento da obesidade infantil: revisão integrativa [Artigo científico de Curso de Graduação, Universidade Católica do Salvador].

Turco, G., Bobbio, T., Reimão, R., Rossini, S., Pereira, H., \& Barros Filho, A. (2013). Quality of life and sleep in obese adolescents. Arquivos de NeuroPsiquiatria, 71, 78-82. DOI: 10.1590/S0004282X2013005000008

Vaz, A. I., Silva, D. I. I., Rego, C. I. I. I. \& Viana, V. I. V. (2010). Determinantes comportamentais em crianças e adolescentes com diagnóstico de obesidade. Alimentação Humana, 16(2).

Viana V, Candeias L, Rego C, Silva D. (2009). Comportamento Alimentar em Crianças e Controlo Parental: Uma Revisão Bibliográfica. Alimentação Humana, 15(1), 9-16.

Viana, V., Guimarães, M. J., Teixeira, M. C., \& Barbosa, M. C. (2003). Aquisição e desenvolvimento de atitudes face à saúde na infância e adolescência. Acta Pediátrica Portuguesa, 34, 277-86. 
Viana, V., \& Sinde, S. (2003). Estilo Alimentar: Adaptação e validação do Questionário Holandês do Comportamento Alimentar. Psicologia, Teoria, Investigação e Prática, 1, 59-71.

Viveiro, C., Brito, S. \& Moleiro, P. (2016). Sobrepeso e obesidade pediátrica: a realidade portuguesa. Revista Portuguesa de Saúde Pública, 37(1), 30-37.

Wardl, J., \& Cooke, L. (2005). The Impact of obesity on psychological well-being. Best Practices \& Research Clinical Endocrinology \& Metabolism, 19(3), 421-40.

Weiss, K. M., Leal, D. B., Assis, M. A. \& Pelegrini, A. (2016). Diagnosty accuracy of anthropometric indicators to predict excess body fat in adolescents aged $11-14$ yeats. Revista Brasileira de Cineantropometria e Desempenho Humano, 18(5), 548-56. http://dx.doi. org/10.5007/1980-0037.2016v18n5p548

World Health Organization. (2020). Noncommunicable diseases: childhood overweight and obesity. https://www.whoint/ news-room/q-a-detail/noncommunicablediseases-childhood-overweight-and-obesity

Ximenes, R. C. C. (Orgs) (2019). Transtornos

Alimentares e Neurociência. Appris. 